\title{
CONFORMALITY IN CONNECTION WITH FUNCTIONS OF TWO COMPLEX VARIABLES
}

\author{
BY \\ EDWARD KASNER
}

1. Introduction. In the theory of functions of two complex variables $z=x+i y, w=u+i v$, the transformations of importance are $Z=Z(z, w)$, $W=W(z, w)$ where $Z$ and $W$ are general analytic functions (power series) such that the jacobian $Z_{z} W_{w}-Z_{w} W_{z}$ is not identically zero. Any pair of such functions may be regarded as a transformation from the points $(x, y, u, v)$ to the points $(X, Y, U, V)$ of a given real cartesian four-space $S_{4}$. Poincaré in his fundamental paper in the Palermo Rendiconti (1907) called any such correspondence a regular transformation. We employ also the term pseudoconformal transformation. The totality of these transformations forms an infinite group $G$. This is not the conformal group of the four-space $S_{4}$ as is the case for the infinite group of analytic functions $Z=Z(z)$ of a single complex variable $z=x+i y$. As a matter of fact, the theorem of Liouville states that the conformal group of the four-space $S_{4}$ is merely the fifteen-parameter group of inversions.

In this paper, we shall obtain several geometric characterizations of this group $G$ of regular transformations. Our main theorem is that the group $G$ of regular (or pseudo-conformal) transformations is characterized by the fact that it leaves invariant the pseudo-angle between any curve $C$ and any hypersurface $H$ at their common point of intersection.

The pseudo-angle may be visualized geometrically as follows. Let a lineal element $C$ and a hypersurface element $H$ intersect in a common point $p$. Rotate the lineal element $C$ about the point $p$ into the hypersurface element $H$ in the unique planar direction (the isoclinal planar direction), which has the property that the angle between any two lineal elements of the rotation is equal to the angle between their orthogonal projections onto the $z$ - (and w-) plane. There is a unique lineal element $C_{1}$ in the hypersurface element $H$, which is the end result of this rotation. Our pseudo-angle is then the actual angle between the initial lineal element $C$ and the terminal lineal element $C_{1}$ of this rotation.

In conclusion we study Picard's sixteen-parameter group, used in the theory of hyperfuchsian functions. The only pseudo-conformal transformations actually conformal in $S_{4}$ constitute a nine-parameter subgroup.

Another geometric interpretation of functions of two complex variables is obtained by using point-pairs (bipoints) in the plane; and this is easily ex-

Presented to the Society, September 11, 1908; also at the Zurich International Congress, 1932 ; received by the editors May 26, 1939. 
tended to $n$ variables by using $n$-points or polygons. See the Bulletin of the American Mathematical Society, vol. 15 (1909), p. 159.

2. Isoclinal and reverse isoclinal planes. Before proving these geometric characterizations of the infinite group $G$, we shall have to consider some preliminary definitions and theorems. A surface $s$ of the four-space $S_{4}$ is given by the two equations $F_{1}(x, y, u, v)=0, F_{2}(x, y, u, v)=0$, where $F_{1}$ and $F_{2}$ are two independent functions of $(x, y, u, v)$. Let $P_{s}(x, y, u, v)$ be any point of the surface $s$. Construct the orthogonal projections $P_{z}(x, y, 0,0)$ and $P_{w}(0,0, u, v)$ (by means of absolutely perpendicular planes) of the point $P_{s}$ on the $z$-and $w$-planes respectively. Thus the surface $s$ induces (1) the correspondence $R_{z w}$ between the points $P_{z}$ and $P_{w}$ of the $z$ - and $w$-planes, (2) the correspondence $R_{z s}$ between the points $P_{z}$ and $P_{s}$ of the $z$-plane and the surface $s$, and (3) the correspondence $R_{w s}$ between the points $P_{w}$ and $P_{s}$ of the $w$-plane and the surface $s$. We call $R_{z w}, R_{z s}, R_{w s}$ the three correspondences associated with the surface $s$. The two correspondences $R_{z s}$ and $R_{w s}$ are the result of orthogonal projections of the points of the surface $s$ onto the $z$ - and $w$-planes. The correspondence $R_{z w}$ is given by the equations $F_{1}(x, y, u, v)=0, F_{2}(x, y, u, v)=0$ of the surface $s$. It is noted that any one of these three correspondences may be degenerate.

Since any orthogonal projection of a plane upon a plane in the four-space $S_{4}$ preserves parallel lines, we find that for a plane $\pi$, each of the three associated correspondences $R_{z w}, R_{z \pi}, R_{w \pi}$ is an affine transformation. Conversely if any one of the three correspondences $R_{z w}, R_{z s}, R_{w s}$ associated with a surface $s$ is an affine transformation, then all three are affine transformations and the surface $s$ is a plane. Of course, all of these statements are equivalent to the fact that a plane of the four-space $S_{4}$ is given by two independent linear equations in the unknowns $(x, y, u, v)$.

For a general plane $\pi$, each of the associated correspondences $R_{z w}, R_{z \pi}, R_{w \pi}$ is an affine transformation. If the associated correspondence $R_{z w}$ is a direct (or reverse) similitude, then $\pi$ is termed an isoclinal plane (or a reverse isoclinal plane). For an isoclinal plane, the correspondences $R_{z \pi}$ and $R_{w \pi}$ are both direct or reverse similitudes according to the choice of the positive sense of rotation of the angle in $\pi$. Similarly for a reverse isoclinal plane $\pi$, the correspondences $R_{z \pi}$ and $R_{w \pi}$ are respectively direct and reverse or reverse and direct similitudes according to the choice of the positive sense of rotation of the angle in $\pi$. Thus for an isoclinal or a reverse isoclinal plane, it is found that under each of the three associated correspondences $R_{z w}, R_{z \pi}, R_{w \pi}$ the angle between any two lines is preserved.

An isoclinal plane may be given by the single complex equation $w=l z+m$, where $l$ and $m$ are arbitrary complex constants; whereas a reverse isoclinal plane may be given by the single complex equation $w=l \bar{z}+m$, where $\bar{z}=x-i y$ is the conjugate of $z=x+i y$. Thus in the totality of $\infty^{6}$ planes of the fourspace $S_{4}$, there are $\infty^{4}$ isoclinal (or reverse isoclinal) planes. These $\infty^{4}$ iso- 
clinal (or reverse isoclinal) planes form a linear system of planes. Through any given point (or in any hyperplane) of the four-space $S_{4}$, there are $\infty^{2}$ isoclinal (or reverse isoclinal) planes. There is one and only one isoclinal (or reverse isoclinal) plane which passes through a given line of the four-space $S_{4}$.

We obtain the following three characterizations of the set of $2 \infty^{4}$ isoclinal and reverse isoclinal planes among the totality of $\infty^{6}$ planes of the four-space $S_{4}$. (1) A plane $\pi$ is an isoclinal or a reverse isoclinal plane if and only if at least one of the associated affine transformations $R_{z w}, R_{z \pi}, R_{w \pi}$ is a similitude. (2) The necessary and sufficient condition that a plane $\pi$ be an isoclinal or a reverse isoclinal plane is that the angle between any line $L$ of $\pi$ and its orthogonal projection $L_{z}$ (or $L_{w}$ ) onto the $z$ - (or w-) plane is constant. This result gives the reason for the term isocline. Let $\phi$ (or $\psi$ ) be the constant angle between any line $L$ of the isoclinal or reverse isoclinal plane $\pi$ and its orthogonal projection $L_{z}$ (or $L_{w}$ ) onto the $z$ - (or $w$-) plane. Then $\phi$ and $\psi$ are complementary angles. (3) A plane $\pi$ is an isoclinal or a reverse isoclinal plane if and only if the maximum and minimum angles between the plane $\pi$ and the $z$ - (or $w$-) plane are equal. The common value of the maximum and minimum angles between the isoclinal or reverse isoclinal plane $\pi$ and the $z$ - (or w-) plane is $\phi$ (or $\psi$ ). Thus an isoclinal or a reverse isoclinal plane makes complementary angles with the $z$ - and w-planes. Also any area in any isoclinal or reverse isoclinal plane is equal to the sum or difference of its orthogonal projections on the $z$ - and $w$-planes. Finally we note that for the isoclinal plane $w=l z+m$ or the reverse isocline plane $w=l \bar{z}+m$, the angle $\phi$ is $\operatorname{arc} \tan |l|$, where $|l|$ denotes the absolute value of $l$.

3 . Conformal and reverse conformal surfaces. The envelope of $\infty^{2}$ isoclinal (or reverse isoclinal) planes is called a conformal surface (or a reverse conformal surface). Upon finding the envelope of the $\infty^{2}$ isoclinal planes $w=l(r, t) z+m(r, t)$ (or of the reverse isoclinal planes $w=l(r, t) \bar{z}+m(r, t)$ ) where $l$ and $m$ are complex functions of the real variables $r$ and $t$, we find that any conformal (or reverse conformal) surface may be given by the single complex equation $w=f(z)$ (or $w=f(\bar{z})$ ), where $f$ is an analytic function of $z$ (or $\bar{z}$ ). From this, it follows that a conformal (or reverse conformal) surface may be given by the two real equations $u=u(x, y), v=v(x, y)$, where $u$ and $v$ are arbitrary real functions of $(x, y)$ which satisfy the Cauchy-Riemann equations $u_{x}=v_{y}, u_{y}=-v_{x}$ (or the reverse Cauchy-Riemann equations $u_{x}=-v_{y}, u_{y}=v_{x}$ ).

From the above facts, it easily follows that the correspondence $R_{z w}$ for a conformal (or reverse conformal) surface $s$ is direct conformal (or reverse conformal). For a conformal surface $s$, the correspondences $R_{z s}$ and $R_{w s}$ are both direct or reverse conformal transformations according to the choice of the positive sense of rotation of the angle in $s$. Similarly for a reverse conformal surface $s$, the correspondences $R_{z s}$ and $R_{w s}$ are respectively direct and reverse or reverse and direct conformal according to the choice of the positive sense of rotation of the angle in $s$. Thus for a conformal (or reverse conformal) sur- 
face $s$, each of the associated correspondences $R_{z w}, R_{z s}, R_{w s}$ preserves the angle between two intersecting curves. Conversely if at least one of the associated correspondences $R_{z w}, R_{z s}, R_{w s}$ of a surface $s$ is conformal (direct or reverse), then all three are conformal (direct or reverse), and $s$ is either a conformal or a reverse conformal surface.

4. Statements of our results. Under the group $G$ of regular transformations, every conformal surface is carried into a conformal surface. On the other hand, every reverse conformal surface is not carried into a reverse conformal surface. $A$ transformation $T$ of the four-space $S_{4}$ is regular if and only if it converts every conformal surface into a conformal surface. The group $G$ of regular transformations preserves the angle and also the sense of rotation between any two intersecting curves contained in a conformal surface. Thus this group $G$ induces the group of direct conformal transformations between the conformal surfaces of the four-space $S_{4}$.

If two intersecting curves $C_{1}$ and $C_{2}$ are tangent to a conformal surface at their common point (or two hypersurfaces $H_{1}$ and $H_{2}$ intersect in a conformal surface), then under the group $G$ of regular transformations, the two curves $C_{1}$ and $C_{2}$ (or the two hypersurfaces $H_{1}$ and $H_{2}$ ) possess the angle between them as the fundamental differential invariant of the first order. On the other hand, two intersecting curves $C_{1}$ and $C_{2}$ not both tangent to a conformal surface (or two hypersurfaces $H_{1}$ and $H_{2}$ not intersecting in a conformal surface) at their common point do not possess any differential invariants of the first order under the infinite group $G$ of regular transformations. This means that under the group $G$ of regular transformations, any two concurrent lineal elements not both contained in an isoclinal surface element (or two concurrent hypersurface elements not intersecting in an isoclinal surface element) can be converted into any other two concurrent lineal elements not both contained in an isoclinal surface element (or any other concurrent two hypersurface elements not intersecting in an isoclinal surface element).

The simplest characterization of the group $G$ of regular transformations is connected with the intersection of a curve and a three-dimensional variety. Let a curve $C$ and a hypersurface $H$ intersect in a point $p$. There is a unique isoclinal plane which passes through the point $p$ and tangent to the curve $C$. Let $C_{1}$ be any curve through the point $p$ which is tangent to this isoclinal plane and to the hypersurface $H$. All such curves $C_{1}$ are tangent to each other at the point $p$. The angle between the curve $C$ and the curve $C_{1}$ is the fundamental differential invariant of the first order between the curve $C$ and the hypersurface $H$. This angle is called the pseudo-angle between the curve $C$ and the hypersurface $H$. A transformation $T$ of the four-space $S_{4}$ is regular if and only if it preserves the pseudo-angle between any curve $C$ and any hypersurface $H$. Thus the infinite group $G$ of regular transformations is characterized by the fact that it leaves invariant the pseudo-angle between every curve $C$ and every hypersurface $H$. 
In the final part of our paper, we shall give a brief discussion of the Picard sixteen-parameter group $G_{16}$ of linear fractional transformations in $w$ and $z$. If a regular transformation $T$ converts $4 \infty^{2}$ isoclinal planes into isoclinal planes, then $T$ carries every isoclinal plane into an isoclinal plane, and therefore $T$ is a linear fractional transformation of the group $G_{16}$. For any other regular transformation $T$, at most $3 \infty^{2}$ isoclinal planes become isoclinal planes.

To prove our theorems, we shall have to consider the lineal elements of the four-space $S_{4}$ which pass through a given point. Any lineal element through a fixed point may be defined by $\left(\rho x^{\prime}, \rho y^{\prime}, \rho u^{\prime}, \rho v^{\prime}\right)$, where $x^{\prime}, y^{\prime}, u^{\prime}, v^{\prime}$ denote the differentials $d x, d y, d u, d v$ respectively, and $\rho$ is any real nonzero factor of proportionality. However, to prove our results we shall find it more convenient to define any real lineal element through a given point by the complex coordinates $\left(\rho z^{\prime}, \rho w^{\prime}\right)$, where $z^{\prime}=x^{\prime}+i y^{\prime}, w^{\prime}=u^{\prime}+i v^{\prime}$, and $\rho$ is any real nonzero factor of proportionality.

5. The necessity of our results. Let $T$ be the regular transformation $Z=Z(z, w), W=W(z, w)$. Let $p(x, y, u, v)$ be a fixed point of the four-space $S_{4}$ and let $P(X, Y, U, V)$ be the transformed point under the regular transformation $T$. Then the special projective transformation between the two bundles of lineal elements through the points $p$ and $P$, which is induced by the regular transformation $T$ is given by the equations

$$
\rho Z^{\prime}=\alpha z^{\prime}+\beta w^{\prime}, \quad \rho W^{\prime}=\gamma z^{\prime}+\delta w^{\prime},
$$

where $\alpha, \beta, \gamma, \delta$ are

$$
\begin{aligned}
\alpha & =\frac{\partial Z}{\partial z}=\frac{1}{2}\left(\frac{\partial}{\partial x}-i \frac{\partial}{\partial y}\right)(X+i Y)=\frac{1}{2}\left(X_{x}+Y_{y}\right)+\frac{i}{2}\left(-X_{y}+Y_{x}\right) \\
& =X_{x}-i X_{y}, \\
\beta & =\frac{\partial Z}{\partial w}=\frac{1}{2}\left(\frac{\partial}{\partial u}-i \frac{\partial}{\partial v}\right)(X+i Y)=\frac{1}{2}\left(X_{u}+Y_{v}\right)+\frac{i}{2}\left(-X_{v}+Y_{u}\right) \\
& =X_{u}-i X_{v}, \\
\gamma & =\frac{\partial W}{\partial z}=\frac{1}{2}\left(\frac{\partial}{\partial x}-i \frac{\partial}{\partial y}\right)(U+i V)=\frac{1}{2}\left(U_{x}+V_{y}\right)+\frac{i}{2}\left(-U_{y}+V_{x}\right) \\
& =U_{x}-i U_{y}, \\
\delta & =\frac{\partial W}{\partial w}=\frac{1}{2}\left(\frac{\partial}{\partial u}-i \frac{\partial}{\partial v}\right)(U+i V)=\frac{1}{2}\left(U_{u}+V_{v}\right)+\frac{i}{2}\left(-U_{v}+V_{u}\right) \\
& =U_{u}-i U_{v} .
\end{aligned}
$$

Any hypersurface is defined by the equation $H(x, y, u, v)=0$, where $H$ is any arbitrary real function of $(x, y, u, v)$. Thus any hypersurface element through the fixed point $p(x, y, u, v)$ is given by 


$$
a z^{\prime}+b w^{\prime}+\bar{a} \bar{z}^{\prime}+\bar{b} \bar{w}^{\prime}=0,
$$

where $a$ and $b$ are

$$
\begin{aligned}
& a=\frac{1}{2}\left(\frac{\partial}{\partial x}-i \frac{\partial}{\partial y}\right) H=\frac{1}{2}\left(H_{x}-i H_{y}\right), \\
& b=\frac{1}{2}\left(\frac{\partial}{\partial u}-i \frac{\partial}{\partial v}\right) H=\frac{1}{2}\left(H_{u}-i H_{v}\right) .
\end{aligned}
$$

From (3) and (4), we see that any real hypersurface element through the fixed point $p$ is defined by the complex coordinates $(\sigma a, \sigma b)$ where $\sigma$ is a real nonzero factor of proportionality.

From (1) and (3), we find that the special projective transformation between the two bundles of hypersurface elements through the fixed points $p$ and $P$, which is induced by the regular transformation $T$, is given by

$$
\sigma a=\alpha A+\gamma B, \quad \sigma b=\beta A+\delta B .
$$

Since the equation of any conformal surface is of the form $w=f(z)$ where $f$ is an analytic function of $z$, there follows from the equations of any regular tranformation $T$

THEOREM 1. Under the group $G$ of regular transformations, every conformal surface is converted into a conformal surface.

Since every conformal surface becomes a conformal surface, it follows that under the group $G$ of regular transformations, every isoclinal surface element is carried into an isoclinal surface element. This is also a consequence of equations (1) upon observing that the equation of any isoclinal surface element through the fixed point $p$ is $w^{\prime}=l z^{\prime}$, where $l$ is an arbitrary complex constant.

Two lineal elements are said to be an isoclinal pair if they are contained in an isoclinal surface element. The condition for an isoclinal pair of lineal elements is

$$
\frac{z_{2}^{\prime}}{z_{1}^{\prime}}=\frac{w_{2}^{\prime}}{w_{1}^{\prime}}=\text { complex constant (not real) }
$$

Two hypersurface elements are said to form an isoclinal pair if they intersect in an isoclinal surface element. The condition for an isoclinal pair of hypersurface elements is

$$
\frac{a_{2}}{a_{1}}=\frac{b_{2}}{b_{1}}=\text { complex constant (not real). }
$$

From equations (1) and (6), we obtain 
THEOREM 2. Two intersecting curves $C_{1}$ and $C_{2}$ which are tangent to a conformal surface at their common point possess the fundamental differential invariant of first order

$$
\operatorname{amp} \frac{z_{2}^{\prime}}{z_{1}^{\prime}}=\operatorname{amp} \frac{w_{2}^{\prime}}{w_{1}^{\prime}} .
$$

This is the angle between the two curves $C_{1}$ and $C_{2}$. It can be written in the real form

$$
\arctan \frac{d x_{1} d y_{2}-d x_{2} d y_{1}}{d x_{1} d x_{2}+d y_{1} d y_{2}}=\arctan \frac{d u_{1} d v_{2}-d u_{2} d v_{1}}{d u_{1} d u_{2}+d v_{1} d v_{2}} .
$$

By equations (5) and (7), we obtain the following dual result:

THEOREM 3. Two hypersurfaces $H_{1}$ and $H_{2}$ which intersect in a conformal surface possess the fundamental differential invariant of first order

$$
\operatorname{amp} \frac{a_{2}}{a_{1}}=\operatorname{amp} \frac{b_{2}}{b_{1}} .
$$

This is the angle between the two hypersurfaces $H_{1}$ and $H_{2}$. It can be written in the real form

$$
\arctan \frac{H_{1 x} H_{2 y}-H_{1 y} H_{2 x}}{H_{1 x} H_{2 x}+H_{1 y} H_{2 y}}=\arctan \frac{H_{1 u} H_{2 v}-H_{1 v} H_{2 u}}{H_{1 u} H_{2 u}+H_{1 v} H_{2 v}} .
$$

Let us now consider the case where two intersecting curves $C_{1}$ and $C_{2}$ are not both tangent to a conformal surface at their common point. In that case, we can convert any non-isoclinal pair of lineal elements $\left(\rho_{1} Z_{1}^{\prime}, \rho_{1} W_{1}^{\prime}\right)$ and $\left(\rho_{2} Z_{2}^{\prime}, \rho_{2} W_{2}^{\prime}\right)$ in to the lineal elements $(1,0)$ and $(0,1)$, which of course are a non-isoclinal pair of lineal elements. The most general transformation of form (1) that will do this is

$$
\rho Z^{\prime}=\rho_{1} Z_{1}^{\prime} z^{\prime}+\rho_{2} Z_{2}^{\prime} w^{\prime}, \quad \rho W^{\prime}=\rho_{1} W_{1}^{\prime} z^{\prime}+\rho_{2} W_{2}^{\prime} w^{\prime} .
$$

This is an admissible transformation since the jacobian $J=\rho_{1} \rho_{2}\left(Z_{1}^{\prime} W_{2}^{\prime}\right.$ $\left.-Z_{2}^{\prime} W_{1}^{\prime}\right)$ is not zero. Hence we have proved that two intersecting curves $C_{1}$ and $C_{2}$ not tangent to a conformal surface at their common point have no differential invariants of the first order. The dual results for hypersurfaces are also valid. Thus we have

Theorem 4. Two intersecting curves $C_{1}$ and $C_{2}$ not both tangent to a conformal surface at their common point (or two hypersurfaces $H_{1}$ and $H_{2}$ which do not intersect in a conformal surface) possess no differential invariants of the first order.

Let $C\left(z^{\prime}, w^{\prime}\right)$ be a given lineal element and $H(a, b)$ a given hypersurface 
element. There is a unique isoclinal surface element which contains the curve $C\left(z^{\prime}, w^{\prime}\right)$. It is given by

$$
\frac{Z^{\prime}}{z^{\prime}}=\frac{W^{\prime}}{w^{\prime}}=\lambda
$$

where $\lambda$ is a complex constant (not real). Upon substituting this into the equation $a Z^{\prime}+b W^{\prime}+\bar{a} \bar{Z}^{\prime}+\bar{b} \bar{W}^{\prime}=0$ of the hypersurface element $H(a, b)$, we find that the lineal element $C_{1}$ of intersection between the isoclinal surface element (13) and the hypersurface element $H(a, b)$ is given by the equation

$$
\frac{Z^{\prime}}{z^{\prime}}=\frac{W^{\prime}}{w^{\prime}}=i\left(\bar{a} \bar{z}^{\prime}+\bar{b} \bar{w}^{\prime}\right) \text {. }
$$

Since, according to Theorem 2 , the angle between the curves $C$ and $C_{1}$ is invariant, we obtain

Theorem 5. A curve $C$ and a hypersurface $H$ which intersect in a common point possess the fundamental differential invariant of first order

$$
\frac{1}{2} \pi-\operatorname{amp}\left(a z^{\prime}+b w^{\prime}\right),
$$

evaluated at the common point. This is called the pseudo-angle between the curve $C$ and the hypersurface $H$. The pseudo-angle represents the angle between the curve $C$ and any curve $C_{1}$ through the point $p$ such that $C$ and $C_{1}$ are tangent to a conformal surface at the point $p$, and $C_{1}$ is tangent to the hypersurface $H$ at the point p. Dually, we find that the pseudo-angle represents the angle between the hypersurface $H$ and any hypersurface $H_{1}$ through the point $p$ such that $H$ and $H_{1}$ intersect in a conformal surface and $H_{1}$ is tangent to the curve $C$ at the point $p$. This pseudo-angle can be written in the real form

$$
\operatorname{arc} \tan \frac{H_{x} d x+H_{y} d y+H_{u} d u+H_{v} d v}{-H_{y} d x+H_{x} d y-H_{v} d u+H_{u} d v} .
$$

The fact that this is the only differential invariant of the first order between a curve $C$ and a hypersurface $H$ which pass through a given point $p$ is an immediate consequence of equations (1) and (5).

6. The sufficiency of our results. Let a general transformation $T$,

(17) $X=(x, y, u, v), \quad Y=Y(x, y, u, v), U=U(x, y, u, v), V=V(x, y, u, v)$,

be given. $T$ is not necessarily a regular transformation. Let $p(x, y, u, v)$ be a fixed point of the four-space $S_{4}$ and let $P(X, Y, U, V)$ be the transformed point under the transformation $T$. Then $T$ induces the following general projective transformation between the two bundles of lineal elements through the points $p$ and $P$ : 


$$
\begin{aligned}
& \rho X^{\prime}=X_{x} x^{\prime}+X_{y} y^{\prime}+X_{u} u^{\prime}+X_{v} v^{\prime}, \\
& \rho Y^{\prime}=Y_{x} x^{\prime}+Y_{y} y^{\prime}+Y_{u} u^{\prime}+Y_{v} v^{\prime}, \\
& \rho U^{\prime}=U_{x} x^{\prime}+U_{y} y^{\prime}+U_{u} u^{\prime}+U_{v} v^{\prime}, \\
& \rho V^{\prime}=V_{x} x^{\prime}+V_{y} y^{\prime}+V_{u} u^{\prime}+V_{v} v^{\prime} .
\end{aligned}
$$

Changing (18) from the real notation $\left(x^{\prime}, y^{\prime}, u^{\prime}, v^{\prime}\right)$ to the complex notation by means of the equations

$$
\begin{array}{rlrl}
Z^{\prime} & =X^{\prime}+i Y^{\prime}, & x^{\prime}=\frac{1}{2}\left(z^{\prime}+\bar{z}^{\prime}\right), & y^{\prime}=\frac{i}{2}\left(\bar{z}^{\prime}-z^{\prime}\right), \\
W^{\prime}=U^{\prime}+i V^{\prime}, & u^{\prime}=\frac{1}{2}\left(w^{\prime}+\bar{w}^{\prime}\right), & v^{\prime}=\frac{i}{2}\left(\bar{w}^{\prime}-w^{\prime}\right),
\end{array}
$$

we find that equations (18) may be written in the compact complex form

$$
\rho Z^{\prime}=\alpha z^{\prime}+\beta w^{\prime}+\phi \bar{z}^{\prime}+\psi \bar{w}^{\prime}, \quad \rho W^{\prime}=\gamma z^{\prime}+\delta w^{\prime}+\chi \bar{z}^{\prime}+\omega \bar{w}^{\prime},
$$

where $\alpha, \beta, \gamma, \delta, \phi, \psi, \chi, \omega$ are given :

$$
\begin{aligned}
& \alpha=\frac{1}{2}\left(\frac{\partial}{\partial x}-i \frac{\partial}{\partial y}\right)(X+i Y)=\frac{1}{2}\left(X_{x}+Y_{y}\right)+\frac{i}{2}\left(-X_{y}+Y_{x}\right), \\
& \beta=\frac{1}{2}\left(\frac{\partial}{\partial u}-i \frac{\partial}{\partial v}\right)(X+i Y)=\frac{1}{2}\left(X_{u}+Y_{v}\right)+\frac{i}{2}\left(-X_{v}+Y_{u}\right), \\
& \gamma=\frac{1}{2}\left(\frac{\partial}{\partial x}-i \frac{\partial}{\partial y}\right)(U+i V)=\frac{1}{2}\left(U_{x}+V_{y}\right)+\frac{i}{2}\left(-U_{y}+V_{x}\right), \\
& \delta=\frac{1}{2}\left(\frac{\partial}{\partial u}-i \frac{\partial}{\partial v}\right)(U+i V)=\frac{1}{2}\left(U_{u}+V_{v}\right)+\frac{i}{2}\left(-U_{v}+V_{u}\right), \\
& \phi=\frac{1}{2}\left(\frac{\partial}{\partial x}+i \frac{\partial}{\partial y}\right)(X+i Y)=\frac{1}{2}\left(X_{x}-Y_{y}\right)+\frac{i}{2}\left(X_{y}+Y_{x}\right), \\
& \psi=\frac{1}{2}\left(\frac{\partial}{\partial u}+i \frac{\partial}{\partial v}\right)(X+i Y)=\frac{1}{2}\left(X_{u}-Y_{v}\right)+\frac{i}{2}\left(X_{v}+Y_{u}\right), \\
& \chi=\frac{1}{2}\left(\frac{\partial}{\partial x}+i \frac{\partial}{\partial y}\right)(U+i V)=\frac{1}{2}\left(U_{x}-V_{y}\right)+\frac{\imath}{2}\left(U_{v}+V_{x}\right), \\
& \omega=\frac{1}{2}\left(\frac{\partial}{\partial u}+i \frac{\partial}{\partial v}\right)(U+i V)=\frac{1}{2}\left(U_{u}-V_{v}\right)+\frac{i}{2}\left(U_{v}+V_{u}\right) .
\end{aligned}
$$

The transformation (20) is thus the general projective transformation (18) between the two bundles of lineal elements through the two points $p$ and $P$.

Let the transformation $T$ carry every conformal surface into a conformal 
surface. Then $T$ must convert every isoclinal surface element into an isoclinal surface element. Hence (20) must carry every equation of the form $w^{\prime}=l z^{\prime}$ into an equation of the same form. For this to be so, we must have

$$
\phi=\psi=\chi=\omega=0 .
$$

These are the double Cauchy-Riemann equations for the two complex functions $X+i Y$ and $U+i V$. Hence these functions must be analytic functions of $z$ and $w$. Thus

TheOREM 6. Any transformation $T$ of the four-space $S_{4}$ which converts every conformal surface into a conformal surface is a regular transformation. Thus the infinite group $G$ of regular transformations is characterized by the fact that it preserves conformal surfaces.

Next we shall prove that the pseudo-angle (the differential invariant (15) or (16)) of Theorem 5 characterizes the infinite group $G$ of regular transformations. Let the transformation $T$ preserve the differential invariant (15) between every lineal element $c\left(z^{\prime}, w^{\prime}\right)$ and every hypersurface element $h(a, b)$ which passes through the common point $p$. Then under $T$ we must have

$$
\frac{a z^{\prime}+b w^{\prime}}{\bar{a} \bar{z}^{\prime}+\bar{b} \bar{w}^{\prime}}=\frac{A Z^{\prime}+B W^{\prime}}{\bar{A} \bar{Z}^{\prime}+\bar{B} \bar{W}^{\prime}}
$$

where the capital letters denote the transformed lineal element $C\left(Z^{\prime}, W^{\prime}\right)$ and the transformed hypersurface element $H(A, B)$.

First we shall show that any isoclinal pair of lineal elements $c_{1}\left(z_{1}^{\prime}, w_{1}^{\prime}\right)$ and $c_{2}\left(z_{2}^{\prime}, w_{2}^{\prime}\right)$ is converted into an isoclinal pair of lineal elements $C_{1}\left(Z_{1}^{\prime}, W_{1}^{\prime}\right)$ and $C_{2}\left(Z_{2}^{\prime}, W_{2}^{\prime}\right)$. Since $c_{1}\left(z_{1}^{\prime}, w_{1}^{\prime}\right)$ and $c_{2}\left(z_{2}^{\prime}, w_{2}^{\prime}\right)$ are contained in an isoclinal surface element, we must have

$$
\frac{z_{2}^{\prime}}{z_{1}^{\prime}}=\frac{w_{2}^{\prime}}{w_{1}^{\prime}}=\lambda,
$$

where $\lambda$ is a fixed non-real complex number. Let us pass any one of the $\infty^{2}$ hypersurface elements $h(a, b)$ through the lineal element $c_{1}\left(z_{1}^{\prime}, w_{1}^{\prime}\right)$. Then under $T$ the transformed hypersurface element $H(A, B)$ must contain the transformed lineal element $C_{1}\left(Z_{1}^{\prime}, W_{1}^{\prime}\right)$. Hence we must have

$$
a z_{1}^{\prime}+b w_{1}^{\prime}+\bar{a} \bar{z}_{1}^{\prime}+\bar{b} \bar{w}_{1}^{\prime}=0, \quad A Z_{1}^{\prime}+B W_{1}^{\prime}+\bar{A} \bar{Z}_{1}^{\prime}+\bar{B} \bar{W}_{1}^{\prime}=0 .
$$

Under the transformation $T$, the pseudo-angle between the lineal element $c_{2}\left(z_{2}^{\prime}, w_{2}^{\prime}\right)$ and any one of the $\infty^{2}$ hypersurface elements $h(a, b)$ through the lineal element $c_{1}\left(z_{1}^{\prime}, w_{1}^{\prime}\right)$ must be equal to the pseudo-angle between the transformed lineal element $C_{2}\left(Z_{2}^{\prime}, W_{2}^{\prime}\right)$ and the corresponding transformed hypersurface element $H(A, B)$. This means that the equation (23) must be valid for these lineal and hypersurface elements. Then because of (24) and 
(25), the equation (23) becomes

$$
\frac{\lambda}{\bar{\lambda}}=\frac{A Z_{2}^{\prime}+B W_{2}^{\prime}}{\bar{A} \bar{Z}_{2}^{\prime}+\bar{B} \bar{W}_{2}^{\prime}} .
$$

This equation must be true for all the $\infty^{2}$ hypersurface elements $H(A, B)$ which pass through the lineal element $C_{1}\left(Z_{1}^{\prime}, W_{1}^{\prime}\right)$.

From this equation, and from the fact that $A Z_{1}^{\prime}+B W_{1}^{\prime}=-\left(\bar{A} \bar{Z}_{1}^{\prime}+\bar{B} \bar{W}_{1}^{\prime}\right)$,

$$
A Z_{2}^{\prime}+B W_{2}^{\prime}=i \rho_{1} \lambda, \quad A Z_{1}^{\prime}+B W_{1}^{\prime}=i \rho_{2},
$$

where $\rho_{1}$ and $\rho_{2}$ are arbitrary real numbers. Let us suppose that $Z_{2}^{\prime} / Z_{1}^{\prime}$ $\neq W_{2}^{\prime} / W_{1}^{\prime}$. From these two equations, we can solve for $A$ and $B$ in terms of the arbitrary real numbers $\rho_{1}$ and $\rho_{2}$. Thence $A$ and $B$ are linear homogeneous functions of $\rho_{1}$ and $\rho_{2}$. This proves that the equation (26) can hold for only $\infty^{1}$ hypersurface elements passing through the lineal element $C_{1}\left(Z_{1}^{\prime}, W_{1}^{\prime}\right)$. This contradicts the fact that the equation (26) must hold for all the hypersurface elements $H(A, B)$ through the lineal element $C_{1}\left(Z_{1}^{\prime}, W_{1}^{\prime}\right)$. Hence we must have

$$
\frac{Z_{2}^{\prime}}{Z_{1}^{\prime}}=\frac{W_{2}^{\prime}}{W_{1}^{\prime}}
$$

This shows that the transformed lineal elements $C_{1}\left(Z_{1}^{\prime}, W_{1}^{\prime}\right)$ and $C_{2}\left(Z_{2}^{\prime}, W_{2}^{\prime}\right)$ must be contained in an isoclinal surface element. Therefore every isoclinal pair of lineal elements is converted by $T$ into an isoclinal pair of lineal elements.

Since any isoclinal pair of lineal elements is carried by $T$ into an isoclinal pair of lineal elements, it follows that $T$ carries every isoclinal surface element into an isoclinal surface element. Hence every conformal surface becomes a conformal surface and the transformation $T$ must therefore be a regular transformation. Thus we have proved

TheOREM 7. Any transformation $T$ of the four-space $S_{4}$ which preserves the pseudo-angle (the differential expression of the first order (15) or (16)) between every curve and every hypersurface evaluated at their common point must be a regular transformation. Thus the infinite group $G$ of regular transformations is characterized by the fact that it leaves invariant the pseudo-angle between every curve and every hypersurface.

7. The Picard sixteen-parameter group $G_{16}$ of linear fractional transformations. In this section, we shall give a characterization of the group $G_{16}$ of the linear fractional transformations in $z$ and $w$

$$
Z=\frac{a_{1} z+b_{1} w+c_{1}}{a z+b w+c}, \quad W=\frac{a_{2} z+b_{2} w+c_{2}}{a z+b w+c} .
$$


Any transformation of the form (29) is a quadric Cremona transformation. It may be considered to be a direct generalization of the Moebius group of circular transformations. Of course, it is not the inversion group of the fourspace $S_{4}$. As a matter of fact, any hypersphere (or any hyperplane) is converted by (29) into a special type of quadric hypersurface.

Under any regular transformation $T$, let us find what isoclinal planes become isoclinal planes. For this to be so, the differential equation $d^{2} w / d z^{2}=0$ must be carried into the differential equation $d^{2} W / d Z^{2}=0$. Hence those isoclinal planes which become isoclinal equations under the regular transformation $T$ must satisfy the equation

$$
\begin{aligned}
\left(Z_{z}+\frac{d w}{d z} Z_{w}\right) & {\left[W_{z z}+2 \frac{d w}{d z} W_{z w}+\left(\frac{d w}{d z}\right)^{2} W_{w w}\right] } \\
& -\left(W_{z}+\frac{d w}{d z} W_{w}\right)\left[Z_{z z}+2 \frac{d w}{d z} Z_{z w}+\left(\frac{d w}{d z}\right)^{2} Z_{w w}\right]=0 .
\end{aligned}
$$

First, if this equation is an identity in $d w / d z$, we find that $Z$ and $W$ must be given by the equations (29). That is, the group $G_{16}$ of linear fractional transformations as given by the equations (29) convert every isoclinal plane into an isoclinal plane.

Next if the above equation is not identically zero, we can solve (30) for $d w / d z$ and obtain at most three differential equations of the form

$$
\frac{d w}{d z}=f(z, w)
$$

where $f$ is an analytic function of $z$ and $w$. Any such differential equation contains $\infty^{2}$ solutions. Thus we have proved

THEоRем 8. If a regular transformation $T$ converts $4 \infty^{2}$ isoclinal planes into isoclinal planes, then every isoclinal plane is converted into an isoclinal plane, and therefore $T$ is a transformation of the group $G_{16}$ of the linear fractional transformations as given by equations (29). Any other regular transformation $T$ converts at most $3 \infty^{2}$ isoclinal planes into isoclinal planes.

It is found that, under the group $G_{16}$ of fractional linear transformations as given by (29), the family of quadric hypersurfaces

$$
a z \bar{z}+b w \bar{w}+\gamma \bar{z} w+\bar{\gamma} z \bar{w}+\delta z+\epsilon w+\bar{\delta} \bar{z}+\bar{\epsilon} \bar{w}+f=0,
$$

where $a, b, f$ are arbitrary real constants and $\gamma, \delta, \epsilon$ are arbitrary complex constants, is converted into itself. The real form of this family of quadric hypersurfaces is

$$
\begin{aligned}
a\left(x^{2}+y^{2}\right)+b\left(u^{2}+v^{2}\right)+2 c_{1}(u x+v y)+2 c_{2}(-u y+v x) \\
+2 d_{1} x+2 d_{2} y+2 e_{1} u+2 e_{2} v+f=0 .
\end{aligned}
$$


There are $\infty^{8}$ hypersurfaces in this family. Every hypersphere (or every hyperplane) of the four-space $S_{4}$ becomes a special quadric hypersurface of the form (32) or (33). Also the intersection of any isoclinal plane with this special quadric hypersurface is a circle. Thus any transformation of the form (29) induces a Moebius circular transformation between the isoclinal planes of the four-space $S_{4}$. In this respect, the group $G_{16}$ of linear fractional transformations in $z$ and $w$ may be regarded as a generalization of the Moebius group of circular transformations to four-space. Also the family of special quadric hypersurfaces (32) or (33) can be considered to be a generalization of the family of circles.

In conclusion I wish to express my thanks to Dr. J. De Cicco for his valuable assistance in writing this paper.

Columbia University, NEW YoRK, N. Y. 